\title{
A Numerical Method Based on Daubechies Wavelet Basis and B-Spline Patches for Elasticity Problems
}

\author{
Yanan Liu and Keqin Din \\ China Special Equipment Inspection and Research Institute, Beijing 100029, China \\ Correspondence should be addressed to Yanan Liu; liuyanan@csei.org.cn
}

Received 11 May 2016; Revised 6 July 2016; Accepted 25 July 2016

Academic Editor: Lihua Wang

Copyright ( 2016 Y. Liu and K. Din. This is an open access article distributed under the Creative Commons Attribution License, which permits unrestricted use, distribution, and reproduction in any medium, provided the original work is properly cited.

\begin{abstract}
The Daubechies (DB) wavelets are used for solving 2D plane elasticity problems. In order to improve the accuracy and stability in computation, the DB wavelet scaling functions in $[0,+\infty)$ comprising boundary scaling functions are chosen as basis functions for approximation. The B-spline patches used in isogeometry analysis method are constructed to describe the problem domain. Through the isoparametric analysis approach, the function approximation and relevant computation based on DB wavelet functions are implemented on B-spline patches. This work makes an attempt to break the limitation that problems only can be discretized on uniform grids in the traditional wavelet numerical method. Numerical examples of 2D elasticity problems illustrate that this kind of analysis method is effective and stable.
\end{abstract}

\section{Introduction}

Wavelet is a powerful mathematical tool in solving many problems in science and engineering. In recent years, there has been an increasing interest in wavelet-based methods due to their successes in some applications, such as mathematical analysis and signal processing. The wavelet-based numerical methods have been developed by many researchers. At present, there are mainly three kinds of wavelet-based numerical methods: wavelet finite element method, wavelet collocation method, and wavelet-Galerkin method. The wavelet finite element method [1-4] is based on traditional FEM. In this method, the scaling functions and wavelet functions in wavelet analysis are used as basis functions to construct the so-called shape functions on elements. In wavelet collocation method [5], the scaling functions and wavelet functions are directly used as basis functions to approximate the unknown functions instead of constructing shape functions as done in the finite element method, and the collocation approach is used for discretization. Based on Daubechies wavelet, some numerical examples for 1- and 2-dimensional model problems show that the wavelet collocation methods are stable and effective for PDE.

The wavelet-Galerkin method [6-13] is the most popular wavelet-based numerical method. Unlike the wavelet collocation method, the Galerkin approach is used for discretization. Although the computational efficiency of Galerkin method is lower than that of collocation method, the accuracy and stability are improved remarkably. The desirable advantage of wavelet is the multiresolution property. Based on the property, the wavelet-based multiscale analysis is easy to be realized [13-19].

Although wavelets have demonstrated potential in numerical simulation, there are still some works to do for engineering application. The traditional wavelets, such as some orthogonality or biorthogonality wavelets, can describe details of problems and perform well in resolving high gradients. However, such wavelets are best suitable for problems that are discretized on uniform grids, a constraint that can be rather restrictive when it comes to modeling problems with complex geometry. By contrast, the finite elements method is very well suited for complex meshes. Some works have been made to use this kind of wavelets to solve problems with general domains $[12,13]$ and the wavelet-based multiscale analysis method can be used to conduct local analysis [13]. But the computational efficiency of this kind of methods still needs to be improved. The second generation wavelets can be constructed on nonuniform grids for numerical simulation $[20,21]$. However, these kinds of wavelets are 
either complex in construction or too simple to simulate complicated deformation.

The isogeometry analysis method [22, 23] developed in recent years presented some new ideas in numerical simulation. In this method, the B-spline functions or nonuniform rational B-spline functions are used to describe the problem geometry and the total solution domain can be divided into many B-spline patches which are similar to the elements in finite element method. Function approximation and relative computation can be implemented on B-spline patches through isoparametric analysis approach. It can be found that the traditional wavelet basis functions are similar to B-spline basis functions in framework that they must be constructed on structure grids. So it is reasonable to introduce the Bspline patches into the problems in which the traditional wavelet basis functions are used. This is an attempt to break the limitation that the traditional wavelet-based numerical methods are only restricted on uniform grids.

In this paper, the Daubechies (DB) wavelet which has orthogonality and compact support is chosen for analysis because of its good performance in numerical simulation. In order to improve the accuracy and stability in computation, the DB wavelet scaling functions in $[0,+\infty)$ which comprise boundary scaling functions are used as basis functions for approximation. The B-spline patches constructed by the Bspline basis functions are used to describe the problem geometry. The function approximation based on DB wavelet basis functions and relevant computations are implemented on Bspline patches through the isoparametric analysis approach. Numerical examples for 2D elasticity problems are given to illustrate the effectiveness of the present method.

\section{The Function Approximation by DB Wavelet Scaling Function}

2.1. The Basic Properties of DB Wavelet. According to the theory of DB wavelet, the so-called scaling function $h(x)$ and wavelet function $g(x)$ of DB wavelet both satisfy two-scaling relation:

$$
\begin{aligned}
& h^{n}(x)=\sum_{j=0}^{2 n-2} p_{j} h^{n}(2 x-j), \\
& g^{n}(x)=\sum_{j=3-2 n}^{1}(-1)^{i} p_{1-j} h^{n}(2 x-j) .
\end{aligned}
$$

Here, the index $n$ denotes the ordinal number of DB wavelet series (DB wavelet with ordinal number $n$ is abbreviated as " $\mathrm{DB}_{n}$ " in the following), and $j$ denotes the place. $p_{j}(j=$ $0,1, \ldots, n-1)$ are called filter coefficients. The supports of scaling function and wavelet function of $\mathrm{DB}$ wavelet are, respectively,

$$
\begin{aligned}
\operatorname{supp} h^{n}(x) & =[0,2 n-1], \\
\operatorname{supp} g^{n}(x+n-1) & =[0,2 n-1] .
\end{aligned}
$$

DB wavelet function $g^{n}(x)$ has $n$ consecutive moments equal to zero. That is vanishing moment $n$ :

$$
\int_{-\infty}^{+\infty} x^{k} g^{n}(x)=0, \quad k=0, \ldots, n-1 .
$$

The ordinal number of DB wavelet series is equal to the number of its vanishing moments. Furthermore, the smoothness of DB wavelet scaling functions and wavelet functions will be improved with the increase of number of vanishing moments.

From translation and dilation of a basic scaling function $h(x)$, we have

$$
h_{i, j}(x)=h\left(2^{-i} x-j\right) .
$$

In the above equation, $i$ and $j$ denote, respectively, the scale and the place in wavelet space. It is obvious that the support of the function $h_{i, j}^{n}(x)$ is

$$
\operatorname{supp} h_{i, j}^{n}=\left[2^{-i} j, 2^{-i}(j+2 n-1)\right] .
$$

According to the principle of multiresolution, the scaling function $h_{i, j}$ can be used to build the wavelet space $V_{i}$ :

$$
\begin{aligned}
V_{i} & =\operatorname{span}\left\{h_{i, j}, j \in Z\right\}, \\
\cdots & \subset V_{i} \subset V_{i+1} \subset V_{i+2} \subset \cdots \\
\bigcap_{i \in Z} V_{i} & =0, \\
\bigcup_{i \in Z} V_{i} & =L^{2}(R) .
\end{aligned}
$$

In addition, the scaling function $h(x)$ can be used to exactly represent polynomial to some degrees. For $\alpha=0$, $1, \ldots, n-1$, we can write

$$
\frac{x^{\alpha}}{\alpha !}=\sum_{k=-\infty}^{+\infty} P_{\alpha}(k) h^{n}(x-k) .
$$

$P_{\alpha}(k)$ are defined by

$$
P_{\alpha}(X)=\sum_{n=0}^{\alpha} \frac{C_{\alpha-n}}{n !} X^{n},
$$

where

$$
C_{m}=\int_{-\infty}^{\infty} \frac{x^{m}}{m !} h^{n}(x) d x
$$

2.2. The $D B$ Wavelet Basis Functions on $[0,+\infty)$. For $\alpha=$ $0,1, \ldots, n-1$, the boundary scaling functions are defined by

$$
\tilde{h}_{\alpha}(x)=\sum_{k=-2 n+2}^{-1} P_{\alpha}(k) h^{n}(x-k) \chi_{[0,+\infty)}(x) .
$$

From the boundary scaling functions $\widetilde{h}_{\alpha}(x)$, we have the formula for all $x$ in $[0,+\infty)$ :

$$
\frac{x^{\alpha}}{\alpha !}=\tilde{h}_{\alpha}(x)+P_{\alpha}(0) h^{n}(x)+P_{\alpha}(1) h^{n}(x-1)+\cdots .
$$



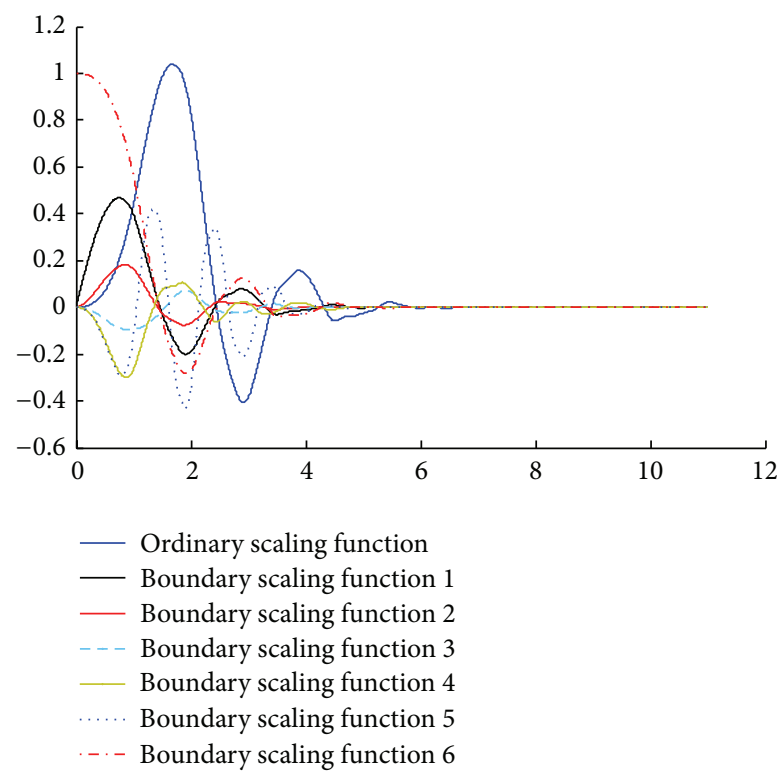

FIGURE 1: Ordinary and boundary scaling functions.

The boundary scaling functions $\widetilde{h}_{\alpha}(x), \alpha=0,1, \ldots, n-1$, are linearly independent and are orthogonal to the functions $h(x-j)$ for $j \geq 0$ (called the ordinary scaling functions on $[0,+\infty)$ ). Figure 1 shows the boundary scaling functions and one of the ordinary scaling functions of DB6 wavelet.

For scale $i$, the function $\widetilde{h}_{\alpha, i}(x)$ is defined as

$$
\widetilde{h}_{\alpha, i}(x)=\widetilde{h}_{\alpha}\left(2^{i} x\right) \text {. }
$$

Then we can define

$$
V_{i}^{[0,+\infty)}=\operatorname{Span}\left\{\left(\widetilde{h}_{\alpha, i}(\cdot)\right)_{\alpha=0, \ldots, n-1},\left(h_{i, j}(\cdot)\right)_{j \geq 0}\right\} .
$$

It is obvious that polynomials up to degree $n-1$ are in $V_{i}^{[0,+\infty)}$.

2.3. The Function Approximation. The function $f(x)$ defined in $[0,+\infty)$ can be approximated in $V_{i}^{[0,+\infty)}$ as

$$
\begin{aligned}
f_{i}(x)=\sum_{\alpha=0}^{n} a_{1, \alpha} \widetilde{h}_{\alpha, i}(x)+\sum_{j=0}^{+\infty} a_{2, j} h_{i, j}(x), & \\
x & \in[0,+\infty), i, j \geq 0 .
\end{aligned}
$$

In the same way, the function $f(x)$ defined in $[0,1]$ can be approximated in a scale as

$$
\begin{aligned}
f_{i}(x)=\sum_{\alpha=0}^{n} a_{1, \alpha} \widetilde{h}_{\alpha, i}(x)+\sum_{j=0}^{2^{i}-1} a_{2, j} h_{i, j}(x), & \\
x & \in[0,1], i, j \geq 0 .
\end{aligned}
$$

It is obvious that the number of basis functions used for approximation in limited interval is finite. For $2 \mathrm{D}$ case, the $2 \mathrm{D}$ tensor product basis functions based on $1 \mathrm{D}$ basis functions can be used for approximation. For example, the function $f(x, y)$ defined in $[0,1] \times[0,1]$ can be approximated as

$$
\begin{aligned}
f(x, y) \approx & f_{i, k}(x, y) \\
= & \sum_{\alpha_{1}=0}^{n-1} \sum_{\alpha_{2}=0}^{n-1} a_{1, \alpha_{1}, \alpha_{2}} \phi_{1, i, \alpha_{1}, k, \alpha_{2}}^{n}(x, y) \\
& +\sum_{\alpha=0}^{n} \sum_{l=0}^{2^{k}-1} a_{2, \alpha, l} \phi_{2, i, \alpha, k, l}^{n}(x, y) \\
& +\sum_{j=0}^{2^{i}-1} \sum_{\alpha=0}^{n-1} a_{3, j, \alpha} \phi_{3, i, j, k, \alpha}^{n}(x, y) \\
& +\sum_{j=0}^{2^{i}-1} \sum_{l=0}^{2^{k}-1} a_{4, j, l} \phi_{4, i, j, k, l}^{n}(x, y),
\end{aligned}
$$

where

$$
\begin{aligned}
\phi_{1, i, \alpha_{1}, k, \alpha_{2}}^{n}(x, y) & =\widetilde{h}_{\alpha_{1}, i}^{n}(x) \widetilde{h}_{\alpha_{2}, k}^{n}(y), \\
\phi_{2, i, \alpha, k, l}^{n}(x, y) & =\widetilde{h}_{\alpha, i}^{n}(x) h_{k, l}^{n}(y), \\
\phi_{3, i, j, k, \alpha}^{n}(x, y) & =h_{i, j}^{n}(x) \widetilde{h}_{\alpha, k}^{n}(y), \\
\phi_{4, i, j, k, l}^{n}(x, y) & =h_{i, j}^{n}(x) h_{k, l}^{n}(y) .
\end{aligned}
$$

The 2D domain occupied by an elastic body is usually irregular. Function approximations in nonrectangular regions are relatively difficult. In this case, those basis functions which influence the given region, $\Omega$, are needed:

$$
\operatorname{supp} \phi \cap \Omega \neq 0 .
$$

\section{Numerical Implementation}

3.1. Format of Approximation. Considering the approximation formula (17), it can be found that the form of approximation by DB wavelet basis functions is the same as that in conventional meshless methods. Scaling functions are similar to shape functions, and $a_{j}$ are similar to variables related to nodes. However, it should be noted that DB wavelet-based method is different from conventional meshless methods in that there is no need to use information of nodes to construct shape functions. We only need to choose proper scaling functions, wavelet functions, and the number of terms. For practical computations, formula (17) can be rewritten as

$$
f_{m}^{n}(x, y)=\sum_{i} a_{i} \phi_{i}(x, y),
$$

where

$$
\phi_{i}(x, y)=h_{m, j}(x) h_{m, k}(y) .
$$

It can be found that the two place indexes $[j, k]$ of $\phi_{i}$ correspond to a serial number $i$. 
3.2. B-Spline Basis Functions for Describing Geometry. Bspline functions are defined recursively starting with piecewise constants $(p=0)$ :

$$
N_{i, 0}(\xi)= \begin{cases}1 & \text { if } \xi_{i} \leq \xi<\xi_{i+1} \\ 0 & \text { otherwise }\end{cases}
$$

For $p=1,2,3, \ldots$, they are defined by

$$
\begin{aligned}
N_{i, p}(\xi)= & \frac{\xi-\xi_{i}}{\xi_{i+p}-\xi_{i}} N_{i, p-1}(\xi) \\
& +\frac{\xi_{i+p+1}-\xi}{\xi_{i+p+1}-\xi_{i+1}} N_{i+1, p-1}(\xi) .
\end{aligned}
$$

Important properties of B-spline basis functions are as follows:

(1) They constitute a partition of unity; that is, $\forall \xi$,

$$
\sum_{i=1}^{n} N_{i, p}(\xi)=1
$$

(2) The support of each $N_{i, p}$ is compact and contained in the interval $\left[\xi_{i}, \xi_{i+p+1}\right]$.

(3) Each basis function is nonnegative; that is, $N_{i, p}(\xi) \geq$ $0, \forall \xi$.

B-spline curves in $R^{d}$ are constructed by taking a linear combination of B-spline basis functions. The coefficients of the basis functions are referred to as control points. These are somewhat analogous to nodal coordinates in finite element analysis. Piecewise linear interpolation of the control points gives the so-called control polygon. In general, control points are not interpolated by B-spline curves. Given $n$ basis functions, $N_{i, p} i=1,2,3, \ldots, n$, and corresponding control points, $B_{i} \in R^{d} i=1,2,3, \ldots, n$, a piecewise-polynomial Bspline curve is given by

$$
C(\xi)=\sum_{i=1}^{n} N_{i, p}(\xi) B_{i}
$$

In $2 \mathrm{D}$ space, the physical coordinate of the curve can be expressed as

$$
\begin{aligned}
& x(\xi)=\sum_{i=1}^{n} N_{i, p}(\xi) x_{i}, \\
& y(\xi)=\sum_{i=1}^{n} N_{i, p}(\xi) y_{i}
\end{aligned}
$$

where $\left(x_{i}, y_{i}\right)$ is the coordinate of control points $B_{i}$.

Given a control net $B_{i, j} \in R^{d}, i=1,2,3, \ldots, n, j=$ $1,2,3, \ldots, m$, a tensor product B-spline surface is defined by

$$
S(\xi, \eta)=\sum_{i=1}^{n} \sum_{j=1}^{m} N_{i, p}(\xi) M_{j, q}(\eta) B_{i, j}
$$

where $N_{i, p} M_{j, q}$ is $2 \mathrm{D}$ B-spline basis function.
3.3. The Isoparametric Analysis Approach Based on B-Spline Patches and DB Wavelet Basis Functions. The DB wavelets scaling functions are good basis functions for approximation. However, the method of approximation introduced in Section 2 is only suited for problems that are discretized on uniform grids. The isogeometry analysis method introduced the B-spline functions to describe problem geometry. The domain can be subdivided into B-Spline patches which are similar to elements in finite element method. The important character of B-spline patches is that they must not be regular in physical space. So they can describe complex geometry more flexibly than uniform grids. Considering the similarity between wavelet basis functions and B-spline functions in framework, this paper introduces the B-spline patches to problems using wavelet numerical method. Through isoparametric analysis approach, the function approximation and relative computations based on wavelet basis functions can be implemented on B-spline patches.

It should be noted that in the classic isogeometry analysis method [1,7] the NURBS (nonuniform rational B-splines) are employed because they can be used to exactly construct many geometric shapes, such as circles and ellipses. In this paper, only ordinary B-splines are used because they can also describe the complex geometry with enough accuracy.

For $1 \mathrm{D}$ case, the approximation in parametric space by wavelet basis functions is as follows:

$$
f(\xi)=\sum_{\alpha=0}^{n} a_{1, \alpha} \tilde{h}_{\alpha}(\xi)+\sum_{j=0}^{m-1} a_{2, j} h(\xi-j), \quad \xi \in(0, m) .
$$

The relationship of physical coordinate and parametric index can be expressed by B-spline basis functions:

$$
x(\xi)=\sum_{i=1}^{m} N_{i, p}(\xi) x_{i},
$$

where $x_{i}$ is the coordinate parameter related to $N_{i, p}$.

For $2 \mathrm{D}$ case, the approximation can be expressed as

$$
\begin{aligned}
f(\xi, \eta)= & \sum_{\alpha_{1}=0}^{n-1} \sum_{\alpha_{2}=0}^{n-1} a_{1, \alpha_{1}, \alpha_{2}} \phi_{1, \alpha_{1}, \alpha_{2}}^{n}(\xi, \eta) \\
& +\sum_{\alpha=0}^{n-1} \sum_{l=0}^{m_{2}-1} a_{2, \alpha, l} \phi_{2, \alpha, l}^{n}(\xi, \eta) \\
& +\sum_{j=0}^{m_{1}-1} \sum_{\alpha=0}^{n-1} a_{3, j, \alpha} \phi_{3, j, \alpha}^{n}(\xi, \eta) \\
& +\sum_{j=0}^{m_{1}-1} \sum_{l=0}^{m_{2}-1} a_{4, j, l} \phi_{4, j, l}^{n}(\xi, \eta) \\
\phi_{1, \alpha_{1}, \alpha_{2}}^{n}(\xi, \eta)= & \widetilde{h}_{\alpha_{1}}^{n}(\xi) \widetilde{h}_{\alpha_{2}}^{n}(\eta), \\
\phi_{2, \alpha, l}^{n}(\xi, \eta)= & \widetilde{h}_{\alpha}^{n}(\xi) h^{n}(\eta-l),
\end{aligned}
$$




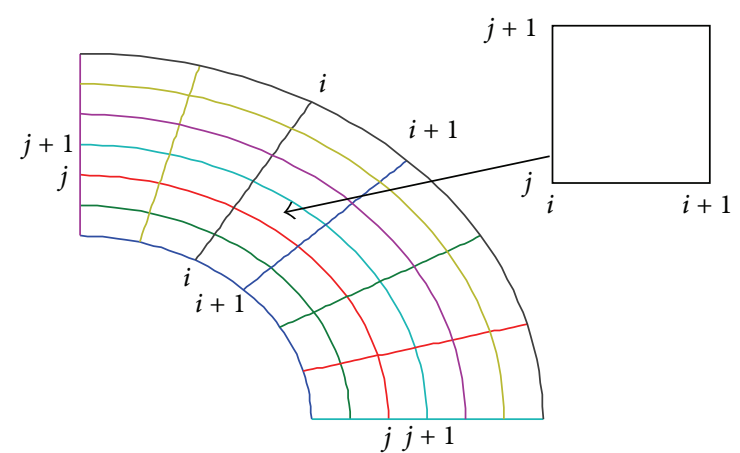

FIGURE 2: Element as parameter span for isoparametric analysis.

$$
\begin{aligned}
\phi_{3, j, \alpha}^{n}(\xi, \eta) & =h^{n}(\xi-j) \widetilde{h}_{\alpha}^{n}(\eta), \\
\phi_{4, j, l}^{n}(\xi, \eta) & =h^{n}(\xi-j) h^{n}(\eta-l) .
\end{aligned}
$$

The relationship of physical space and parametric space can be expressed by $2 \mathrm{D}$ tensor product $\mathrm{B}$-spline basis functions:

$$
\begin{aligned}
& x(\xi, \eta)=\sum_{i=1}^{m_{1}} \sum_{j=1}^{m_{2}} N_{i, p}(\xi) M_{j, q}(\eta) x_{i j}, \\
& y(\xi, \eta)=\sum_{i=1}^{m_{1}} \sum_{j=1}^{m_{2}} N_{i, p}(\xi) M_{j, q}(\eta) y_{i j},
\end{aligned}
$$

where $x_{i j}$ and $y_{i j}$ are, respectively, the coordinate components related to the $2 \mathrm{D} \mathrm{B}$-spline basis functions $N_{i, p} M_{j, q}$. The relevant first-order derivative can be expressed as

$$
\left(\begin{array}{l}
\frac{d f}{d x} \\
\frac{d f}{d y}
\end{array}\right)=\left(\begin{array}{ll}
\frac{d x}{d \xi} & \frac{d y}{d \xi} \\
\frac{d x}{d \eta} & \frac{d y}{d \eta}
\end{array}\right)^{-1}\left(\begin{array}{c}
\frac{d f}{d \xi} \\
\frac{d f}{d \eta}
\end{array}\right) .
$$

Based on the above approximation method, the relative numerical computation can be implemented on "elements" which are taken to be the parameter spans, namely, $[i, i+1] \times$ $[j, j+1]$. Figure 2 illustrates a standard biunit parent element and its image in physical space.

3.4. The Method of Discretization. The equations for the $2 \mathrm{D}$ elasticity problem are expressed as follows:

$$
\begin{aligned}
& \sigma_{i j, j}+b_{i}=0 \text { in } \Omega \\
& \sigma_{i j} n_{j}=\bar{t}_{i} \text { on } \Gamma_{t} \\
& u_{i}=\bar{u}_{i} \text { on } \Gamma_{u} \\
& i, j \in(1,2),
\end{aligned}
$$

where $\sigma_{i j}$ is the stress tensor, $b_{i}$ is the body force, $\bar{t}_{i}$ and $\bar{u}_{i}$ are, respectively, the prescribed tension and displacement, and $n_{j}$ is the unit outward normal to boundary. In general, collocation methods and Galerkin methods are dominant in discretization. In this paper, the Galerkin approach is adopted. Consider the virtual displacement principle

$$
\int_{\Omega}\left(\sigma_{i j, j}+b_{i}\right) \delta u_{i} d \Omega+\int_{\Gamma_{t}}\left(\sigma_{i j} n_{j}-\bar{t}_{i}\right) \delta u_{i} d \Gamma=0
$$

$\delta u_{i}$ is the variation of real displacement. From formula (38), the weak form can be written as

$$
\int_{\Omega} \delta e_{i j} \sigma_{i j} d \Omega=-\int_{\Gamma_{t}} \delta u_{i} \bar{t}_{i} d \Gamma+\int_{\Omega} \delta u_{i} b_{i} d \Omega
$$

where $\delta u_{i}$ vanishes and $u_{i}=\bar{u}_{i}$ on $\Gamma_{u}$.

In $2 \mathrm{D}$ problems, formula (30) can be used for the approximation of the two displacement field functions: $u$ and $v$. For simplification, the approximation formula can be written as

$$
\begin{aligned}
& u(\xi, \eta)=\sum_{i} a_{u i} \phi_{u i}(\xi, \eta), \\
& v(\xi, \eta)=\sum_{j} a_{v j} \phi_{v j}(\xi, \eta),
\end{aligned}
$$

where $a_{u i}$ and $a_{v j}$ are unknown variables. Then we can obtain the discrete equations by introducing the approximation formula (40) into formula (39). Eventually, a group of linear algebraic equations can be obtained:

$$
\mathbf{K a}=\mathbf{f} \text {, }
$$

where

$$
\begin{aligned}
\mathbf{a} & =\left[a_{u 1}, a_{u 2}, \ldots, a_{v 1}, a_{v 2}, \ldots\right]^{T}, \\
\mathbf{K} & =\int_{\Omega} \mathbf{B}^{T} \mathbf{D B} d \Omega, \\
\mathbf{f}^{T} & =\int_{\Omega} \boldsymbol{\varphi}^{T} \mathbf{b} d \Omega+\int_{\Gamma_{t}} \boldsymbol{\varphi}^{T} \overline{\mathbf{t}} d \Gamma .
\end{aligned}
$$

D is the $2 \mathrm{D}$ elasticity matrix:

$$
\mathbf{D}=\frac{E_{0}}{\left(1-v_{0}^{2}\right)}\left[\begin{array}{ccc}
1 & v & 0 \\
\nu & 1 & 0 \\
0 & 0 & \frac{1-v}{2}
\end{array}\right]
$$

Plane stress is as follows:

$$
\begin{aligned}
E_{0} & =E, \\
\nu_{0} & =\nu .
\end{aligned}
$$


Plane strain is as follows:

$$
\begin{aligned}
E_{0} & =\frac{E}{1-v^{2}}, \\
\nu_{0} & =\frac{v}{1-\nu}, \\
\mathbf{B} & =\mathbf{L} \boldsymbol{\varphi} \\
\mathbf{L} & =\left[\begin{array}{cc}
\frac{\partial}{\partial x} & 0 \\
0 & \frac{\partial}{\partial y} \\
\frac{\partial}{\partial y} & \frac{\partial}{\partial x}
\end{array}\right], \\
\boldsymbol{\varphi} & =\left[\begin{array}{cccccc}
\phi_{u 1} & \phi_{u 2} & \cdots & 0 & 0 & \cdots \\
0 & 0 & \cdots & \phi_{v 1} & \phi_{v 2} & \cdots
\end{array}\right] .
\end{aligned}
$$

Here, $\mathbf{K}$ and $\mathbf{f}$ are stiffness matrix and load vector, respectively. In addition, the derivative in formula (47) should be evaluated using formula (36). All relevant computation should be performed on parametric space.

\section{Numerical Examples}

In this part, numerical examples for some 2D plain elasticity problems are presented using the analysis method introduced above. The results are compared with those calculated by finite element method or analytical results to show the validity of the proposed method. For simplification, the units are omitted in this paper. In addition, the DB6 wavelet is used in all examples because it performs better in accuracy and efficiency than other DB wavelets.

4.1. Solid Circular Cylinder Subjected to Internal Pressure Loading. Solid circular cylinder subjected to constant internal pressure loading is considered in this example. Plane strain conditions are assumed to hold in the axial direction. The problem specification is shown in Figure 3. The exact solution for the case with constant pressure is as follows:

$$
\begin{aligned}
u_{r} & =\frac{1}{E} \frac{P R_{i}^{2}}{R_{o}^{2}-R_{i}^{2}}\left((1-v) r+\frac{R_{o}^{2}(1+v)}{r}\right), \\
\sigma_{r} & =\frac{P R_{i}^{2}}{R_{o}^{2}-R_{i}^{2}}\left(1-\frac{R_{o}^{2}}{r^{2}}\right), \\
\sigma_{\theta} & =\frac{P R_{i}^{2}}{R_{o}^{2}-R_{i}^{2}}\left(1+\frac{R_{o}^{2}}{r^{2}}\right) .
\end{aligned}
$$

The three meshes used in the analysis are shown in Figure 4 . They are constructed by the cubic B-spline functions. The displacement and stress results are presented in Table 1. The pressure is set to $p=1$ and material constants are given by $E=1$ and $v=0.3$. It can be found that the numerical results are close to the analytical results even with the first (coarsest) mesh. It is also obvious that the computational accuracy is improved with finer meshes.

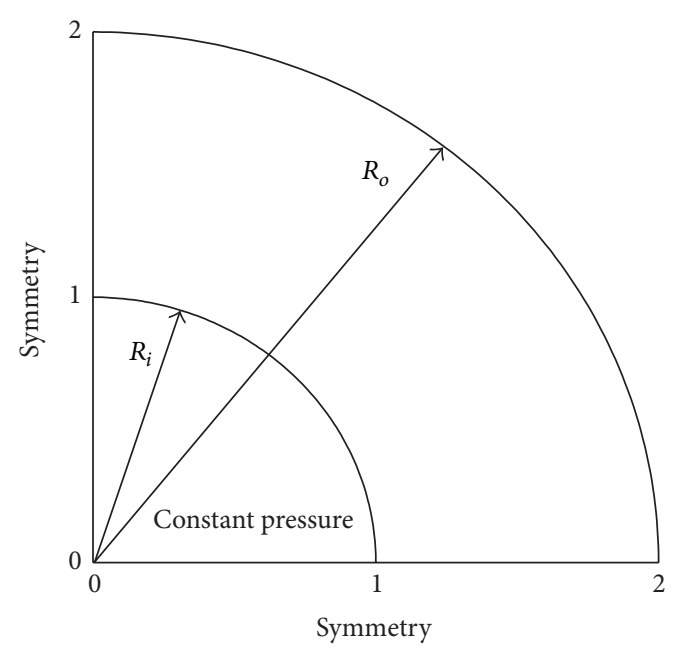

FIGURE 3: Quarter of a thick cylinder under constant internal pressure.

TABLE 1: The comparison of numerical and analytical results.

\begin{tabular}{lcccc}
\hline Scale & \multicolumn{2}{c}{$u_{r}$} & \multicolumn{2}{c}{$\sigma_{\theta}$} \\
& Outer & Internal & Outer & Internal \\
\hline Mesh 1 & 1.325 & 1.961 & 0.6687 & 1.7091 \\
Mesh 2 & 1.330 & 1.964 & 0.6672 & 1.6761 \\
Mesh 3 & 1.331 & 1.965 & 0.6684 & 1.6733 \\
Analytical & 1.3333 & 1.9667 & 0.6667 & 1.6667 \\
\hline
\end{tabular}

4.2. Infinite Plate with a Circle Hole. An infinite plate with a central circular hole as shown in Figure 5 is considered. The plate is subjected to uniform unit traction along the $x$-axis at infinity. Symmetry of the problem is exploited to model only a quadrant of the analysis domain. The radius of the hole is set to be 0.25 and the length and width of the plate are set to be 1 . The exact solutions for this problem are

$$
\begin{aligned}
\sigma_{x} & =1-\frac{a^{2}}{r^{2}}\left(\frac{3}{2} \cos 2 \theta+\cos 4 \theta\right)+\frac{3 a^{4}}{2 r^{4}} \cos 4 \theta, \\
\sigma_{x y} & =-\frac{a^{2}}{r^{2}}\left(\frac{1}{2} \sin 2 \theta+\sin 4 \theta\right)+\frac{3 a^{4}}{2 r^{4}} \sin 4 \theta, \\
\sigma_{y} & =-\frac{a^{2}}{r^{2}}\left(\frac{1}{2} \cos 2 \theta-\cos 4 \theta\right)-\frac{3 a^{4}}{2 r^{4}} \cos 4 \theta .
\end{aligned}
$$

The exact solutions are used to compute the tractions on the traction boundaries (right and top boundaries) and symmetry boundary conditions are applied on the bottom and left edges while the inner edges are traction-free. The three meshes used in this analysis are shown in Figure 6. They are also constructed by the cubic B-spline functions.

Figure 7 shows the stress value $\left(\sigma_{x}\right)$ along the left edge obtained using different meshes. It can be found that the numerical results agree well with the analytical results even using the coarsest mesh. Table 2 shows the comparison of convergent results of stress $\left(\sigma_{x}\right)$ computed by present method and analytical results at different points in the stress 

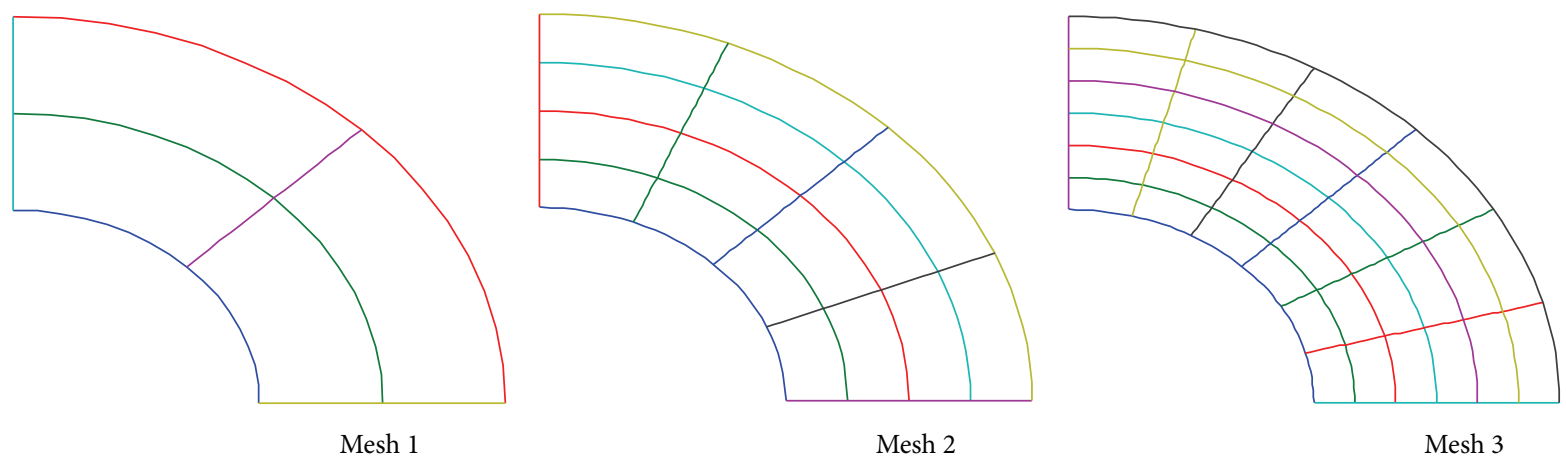

FIGURE 4: Cylinder under internal pressure. Meshes for computation.

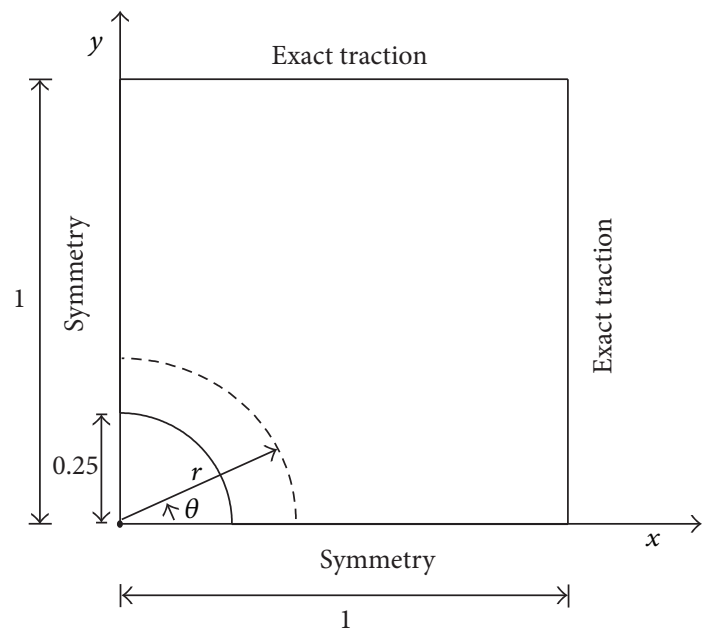

FIgURE 5: Quarter of a panel with a circle hole at its center.

TABLE 2: The comparison of convergent results of stress $\left(\sigma_{x}\right)$ in stress concentration zone.

\begin{tabular}{lcc}
\hline Coordinate $(x, y)$ & $\begin{array}{c}\sigma_{x} \\
\text { Wavelet method } \\
\text { (convergent results) }\end{array}$ & Analytical solution \\
\hline$(0.0,0.25)$ & 3.0107 & 3.0 \\
$(0.0,0.277)$ & 2.4115 & 2.4025 \\
$(0.0488,0.2452)$ & 2.7391 & 2.7389 \\
$(0.0579,0.2725)$ & 2.2156 & 2.2169 \\
\hline
\end{tabular}

concentration zone. The error at every point is very small. Table 3 displays the comparison of the stress concentration factor computed using different meshes.

The plot for strain energy error is shown in Figure 8. The $x$-axis represents the number of degrees of freedom. The convergence of this method is evident. The above numerical results demonstrate that the present method is stable and effective for problems with general domains.

4.3. Plate with a Square Hole. Figure 9 depicts quarter of a panel with a square hole of $a=0.25$ at its center. The uniform
TABLE 3: The comparison of the stress concentration factors computed in different scales.

\begin{tabular}{lc}
\hline Scale & Stress concentration factor \\
\hline Mesh 1 & 3.0794 \\
Mesh 2 & 3.0251 \\
Mesh 3 & 3.0168 \\
Convergence & 3.0107 \\
\hline
\end{tabular}

TABLE 4: The comparison of stress values $\left(\sigma_{x}\right)$ at singular point.

\begin{tabular}{lc}
\hline Scale & Stress $\left(\sigma_{x}\right)$ \\
\hline Mesh 1 & 4.1106 \\
Mesh 2 & 5.6188 \\
Mesh 3 & 8.4542 \\
FE method & 12.386 \\
\hline
\end{tabular}

tension $p=1$ in the horizontal direction is applied on the left and right edges of the panel.

Three meshes used in this analysis are shown in Figure 10. The stress results $\left(\sigma_{x}\right)$ along $y=0.25$ are shown in Figure 11. Table 4 displays the stress values $\left(\sigma_{x}\right)$ at singular point, where the results of FEM are computed using more than 22000 elements of plain eight nodes. It can be found that the present method can catch the singular character with relatively less elements. The plot for strain energy error is shown in Figure 12. The $x$-axis represents the number of degrees of freedom. The strain energy obtained by FEM is used for computing error. It can be observed that the present method exhibits good convergence rates and for a given number of degrees of freedom, the errors are much smaller compared with the traditional wavelet-Galerkin methods. These numerical results demonstrate that the present method can also effectively deal with problems with singularity.

4.4. Semi-Infinite Plate under a Normal Concentration Force. A semi-infinite plate under a normal concentration force $P$ at its boundary is considered. The computation model of this 

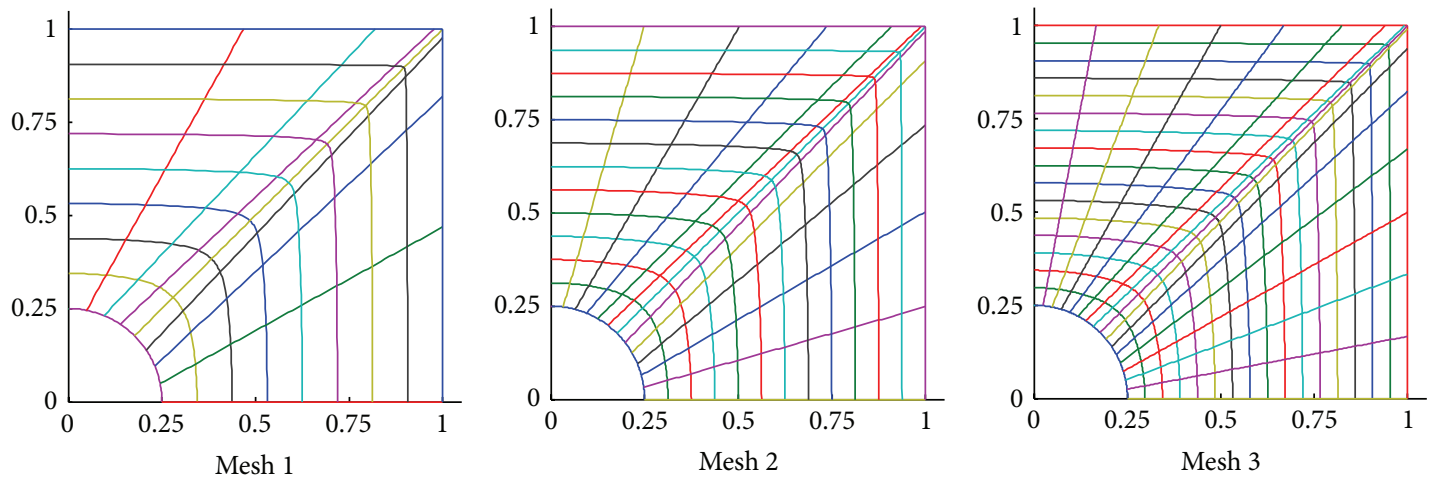

Figure 6: Panel with circle hole. Meshes for computation.

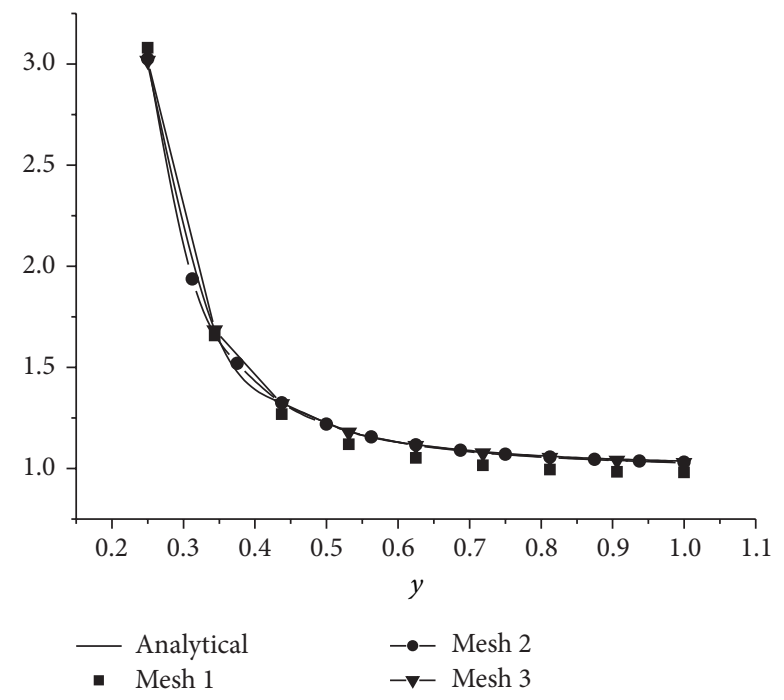

FIgURE 7: Stress $\left(\sigma_{x}\right)$ along $y$-axis.

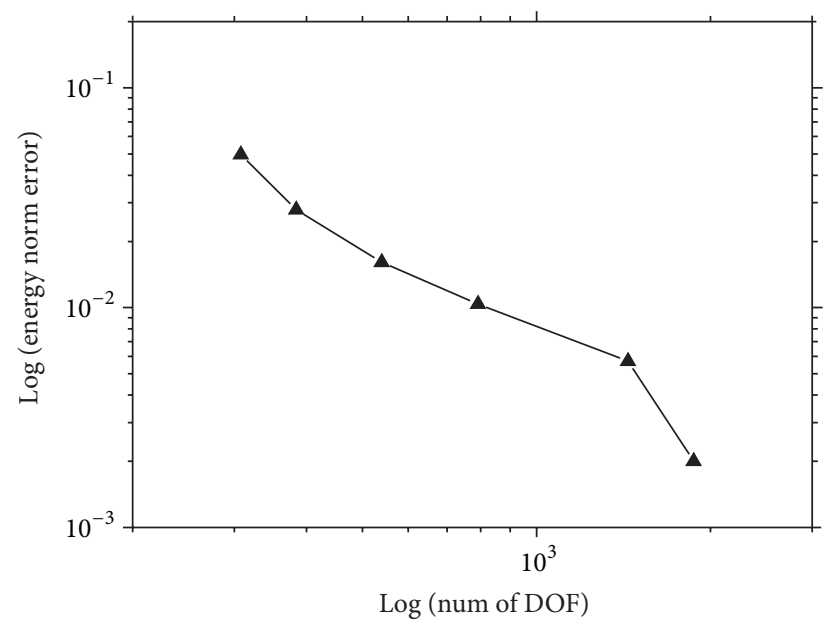

FIGURE 8: Plot of error in strain energy with respect to the number of degrees of freedom.

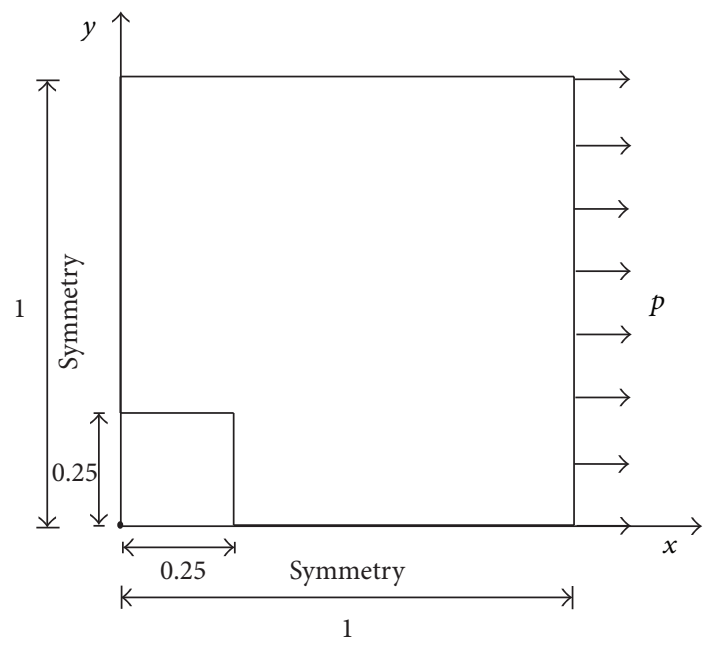

FIGURE 9: Quarter of a panel with a square hole at its center.

problem is shown in Figure 13. The radius of the semicircle in the model is $r=1$. The exact solutions for this problem are

$$
\begin{gathered}
\sigma_{x}=-\frac{2 P}{\pi} \frac{x^{2} y}{\left(x^{2}+y^{2}\right)^{2}}, \\
\sigma_{y}=-\frac{2 P}{\pi} \frac{y^{3}}{\left(x^{2}+y^{2}\right)^{2}}, \\
\sigma_{x y}=-\frac{2 P}{\pi} \frac{x y^{2}}{\left(x^{2}+y^{2}\right)^{2}} .
\end{gathered}
$$

The exact solutions are used to compute the tractions on the traction boundaries (semicircle boundaries) while the other edges are traction-free.

Two meshes used in this analysis are shown in Figure 14. The stress results $\left(\sigma_{y}\right)$ along $x=0$ are shown in Figure 15. It can be found that the accuracy of stress results with two kinds of meshes is good. Furthermore, the stress results obtained using mesh 2 are close to analytical results even near the 

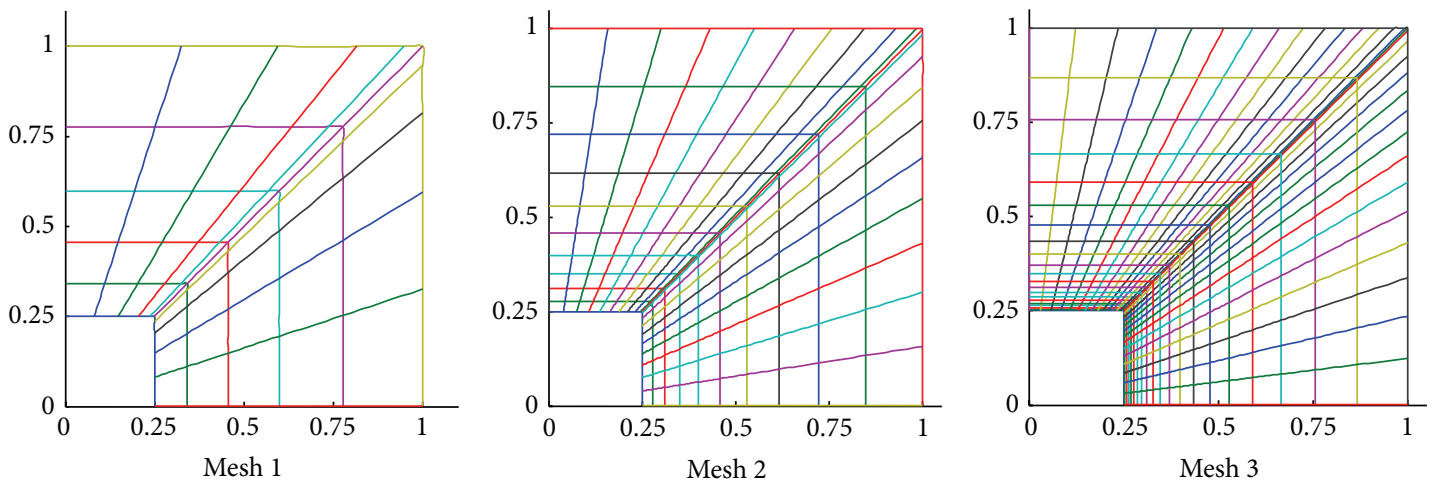

Figure 10: Panel with square hole. Meshes for computation.

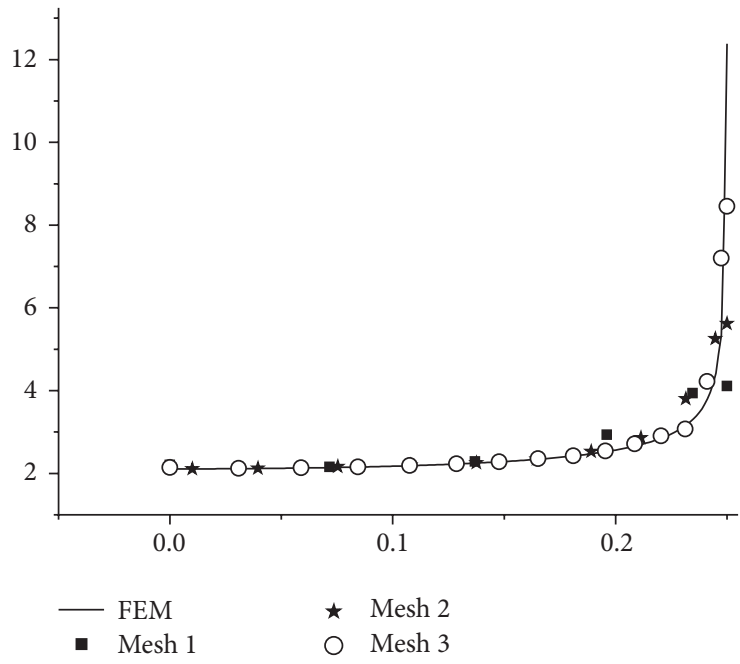

Figure 11: Stress $\left(\sigma_{x}\right)$ along $y=0.25$.

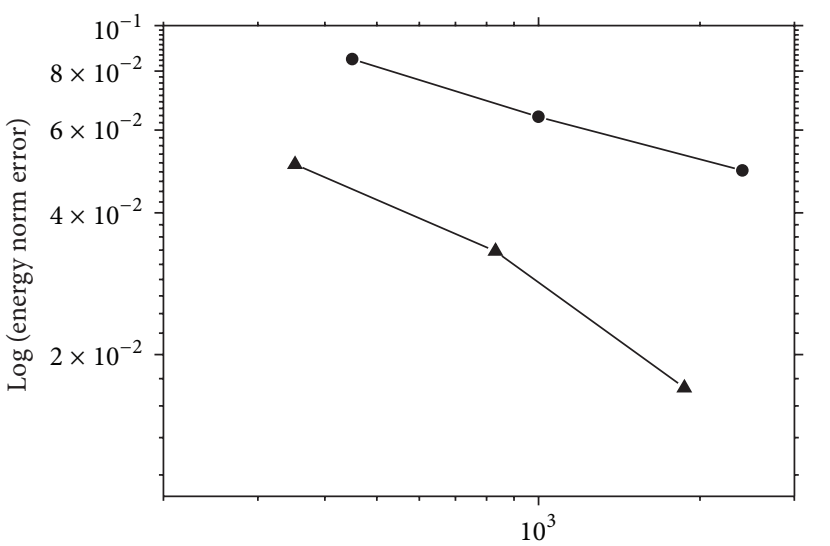

$\log ($ num of DOF)

-4- Present method

-- Traditional method

FIGURE 12: Plot of error in strain energy for problem of a plate with a square hole.

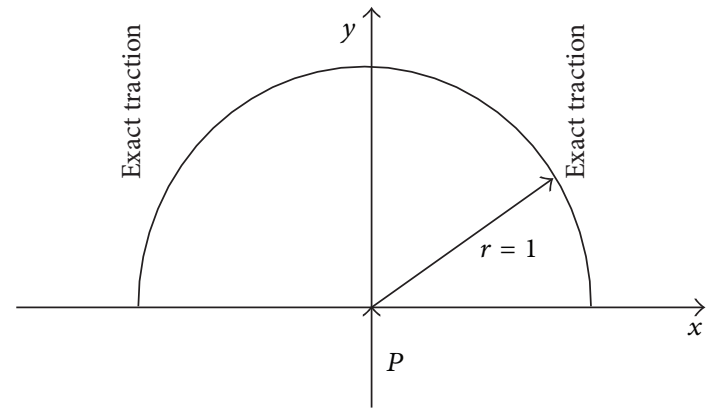

FIgURE 13: The computation model for semi-infinite plate under a normal concentration force.

singular point. The results in this example further prove that the present method can effectively analyze the problems with singularity.

\section{Conclusion}

In this paper, the $\mathrm{DB}$ wavelet scaling functions in $[0,+\infty)$ which comprise edge scaling functions are chosen as basis functions for approximation. The B-spline patches are constructed to describe the problem domain. Through the isoparametric analysis approach, the function approximation and relevant computation based on DB wavelet basis functions are implemented on B-spline patches. Compared with the traditional wavelet numerical methods which rely on uniform grids, this present method is flexible and easy to work with for numerical analysis. Numerical examples of $2 \mathrm{D}$ elasticity problems illustrate that this kind of analysis method is effective and stable. Furthermore, this method gives a thought to perform the traditional wavelet-based numerical analysis on nonuniform grids.

\section{Competing Interests}

The authors declare that they have no competing interests. 


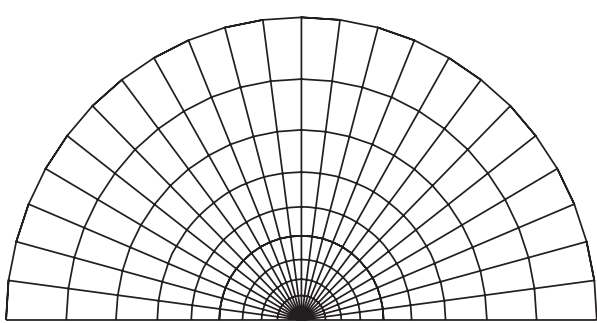

Mesh 1

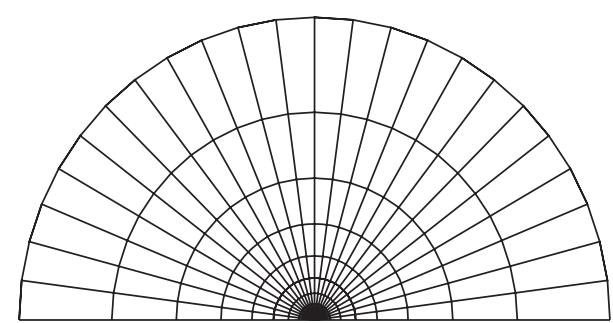

Mesh 2

FIGURE 14: The meshes used for computation.

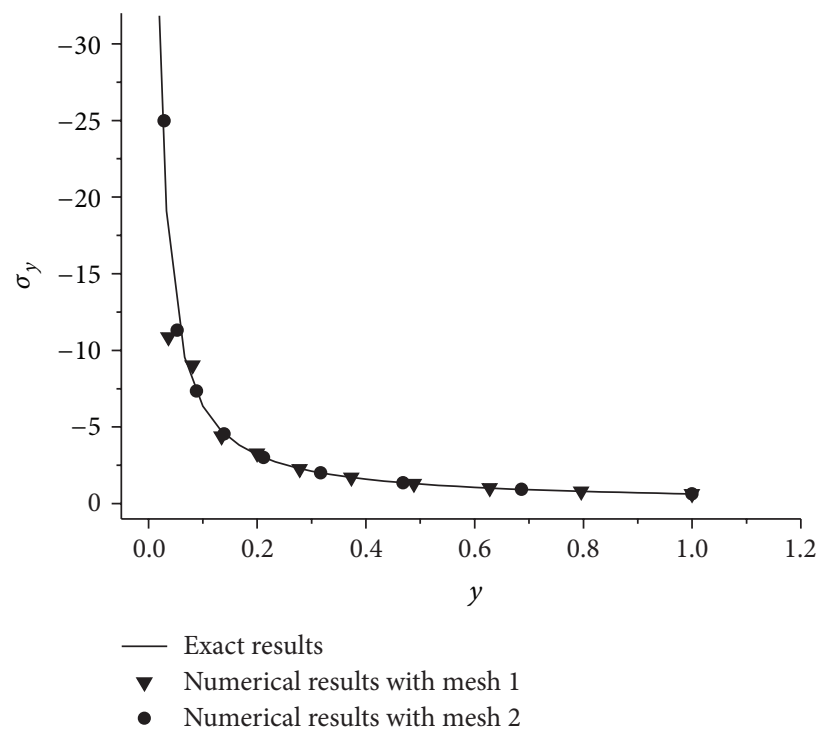

FIGURE 15: Stress $\left(\sigma_{y}\right)$ along $x=0$.

\section{Acknowledgments}

This paper was supported by the National Natural Science Foundation of China (11272358).

\section{References}

[1] J. Ko, A. J. Kurdila, and M. S. Pilant, "A class of finite element methods based on orthonormal, compactly supported wavelets," Computational Mechanics, vol. 16, no. 4, pp. 235-244, 1995.

[2] X. Chen, S. Yang, J. Ma, and Z. He, "The construction of wavelet finite element and its application," Finite Elements in Analysis and Design, vol. 40, no. 5-6, pp. 541-554, 2004.

[3] A. J. Kurdila, T. Sun, P. Grama, and J. Ko, "Affine fractal interpolation functions and wavelet-based finite elements," Computational Mechanics, vol. 17, no. 3, pp. 169-185, 1995.

[4] J.-G. Han, W.-X. Ren, and Y. Huang, "A spline wavelet finiteelement method in structural mechanics," International Journal for Numerical Methods in Engineering, vol. 66, no. 1, pp. 166-190, 2006.

[5] S. Bertoluzza and G. Naldi, "A wavelet collocation method for the numerical solution of partial differential equations," Applied and Computational Harmonic Analysis, vol. 3, no. 1, pp. 1-9, 1996.

[6] K. Amaratunga, J. R. Williams, S. Qian, and J. Weiss, "WaveletGalerkin solutions for one-dimensional partial differential equations," International Journal for Numerical Methods in Engineering, vol. 37, no. 16, pp. 2703-2716, 1994.

[7] K. Amaratunga and J. R. Williams, "Wavelet-Galerkin solution of boundary value problems," Archives of Computational Methods in Engineering, vol. 4, no. 3, pp. 243-285, 1997.

[8] A. R. Díaz, "A wavelet-Galerkin scheme for analysis of largescale problems on simple domains," International Journal for Numerical Methods in Engineering, vol. 44, no. 11, pp. 1599-1616, 1999.

[9] J. M. Restrepo and G. K. Leaf, "Wavelet-Galerkin discretization of hyperbolic equations," Journal of Computational Physics, vol. 122, no. 1, pp. 118-128, 1995.

[10] S. M. Gomes and E. Cortina, "Convergence estimates for the wavelet Galerkin method," SIAM Journal on Numerical Analysis, vol. 33, no. 1, pp. 149-161, 1996.

[11] Y. Liu, F. Qin, Y. Liu, and Z. Cen, "A Daubechies waveletbased method for elastic problems," Engineering Analysis with Boundary Elements, vol. 34, no. 2, pp. 114-121, 2010.

[12] Y. Liu, F. Qin, Y. H. Liu, and Z. Cen, "The 2D large deformation analysis using Daubechies wavelet," Computational Mechanics, vol. 45, no. 2-3, pp. 179-187, 2010.

[13] Y. Liu, Y. H. Liu, and Z. Cen, "Multi-scale Daubechies waveletbased method for 2-D elastic problems," Finite Elements in Analysis and Design, vol. 47, no. 4, pp. 334-341, 2011.

[14] O. V. Vasilyev, S. Paolucci, and M. Sen, "A multilevel wavelet collocation method for solving partial differential equations in a finite domain," Journal of Computational Physics, vol. 120, no. 1, pp. 33-47, 1995.

[15] O. V. Vasilyev and S. Paolucci, "A dynamically adaptive multilevel wavelet collocation method for solving partial differential equations in a finite domain," Journal of Computational Physics, vol. 125, no. 2, pp. 498-512, 1996.

[16] O. V. Vasilyev and N. K. Kevlahan, "An adaptive multilevel wavelet collocation method for elliptic problems," Journal of Computational Physics, vol. 206, no. 2, pp. 412-431, 2005.

[17] Y. Y. Kim and G.-W. Jang, "Hat interpolation wavelet-based multi-scale Galerkin method for thin-walled box beam analysis," International Journal for Numerical Methods in Engineering, vol. 53, no. 7, pp. 1575-1592, 2002.

[18] J. E. Kim, G.-W. Jang, and Y. Y. Kim, "Adaptive multiscale wavelet-Galerkin analysis for plane elasticity problems and its applications to multiscale topology design optimization," 
International Journal of Solids and Structures, vol. 40, no. 23, pp. 6473-6496, 2003.

[19] G.-W. Jang, J. E. Kim, and Y. Y. Kim, "Multiscale Galerkin method using interpolation wavelets for two-dimensional elliptic problems in general domains," International Journal for Numerical Methods in Engineering, vol. 59, no. 2, pp. 225-253, 2004.

[20] K. Amaratunga and J. E. Castrillon-Candas, "Surface wavelets: a multiresolution signal processing tool for 3D computational modelling," International Journal for Numerical Methods in Engineering, vol. 52, no. 3, pp. 239-271, 2001.

[21] K. Amaratunga and R. Sudarshan, "Multiresolution modeling with operator-customized wavelets derived from finite elements," Computer Methods in Applied Mechanics and Engineering, vol. 195, no. 19-22, pp. 2509-2532, 2006.

[22] T. J. Hughes, J. A. Cottrell, and Y. Bazilevs, "Isogeometric analysis: CAD, finite elements, NURBS, exact geometry and mesh refinement," Computer Methods in Applied Mechanics and Engineering, vol. 194, no. 39-41, pp. 4135-4195, 2005.

[23] J. A. Cottrell, T. J. R. Hughes, and A. Reali, "Studies of refinement and continuity in isogeometric structural analysis," Computer Methods in Applied Mechanics and Engineering, vol. 196, no. 41-44, pp. 4160-4183, 2007. 


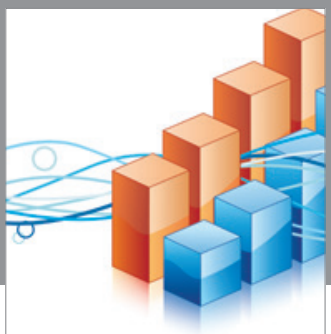

Advances in

Operations Research

vatem alat4

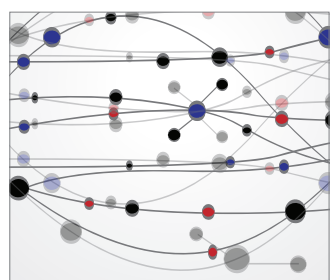

\section{The Scientific} World Journal
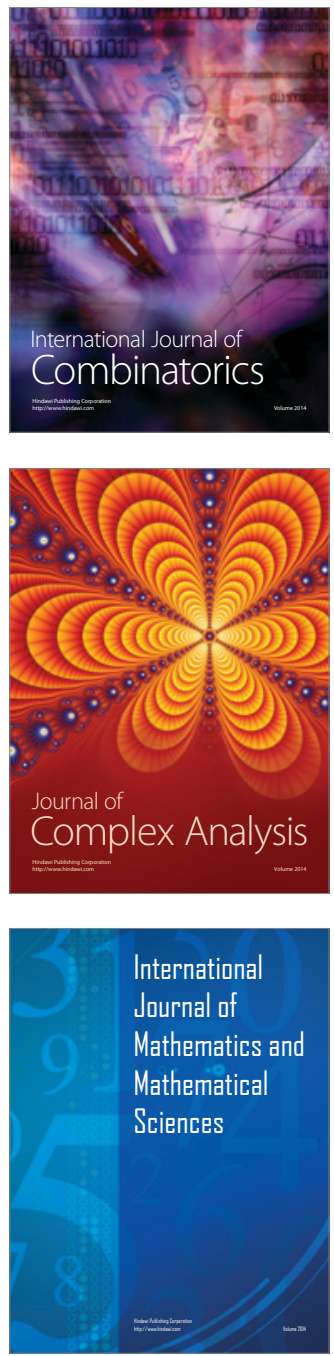
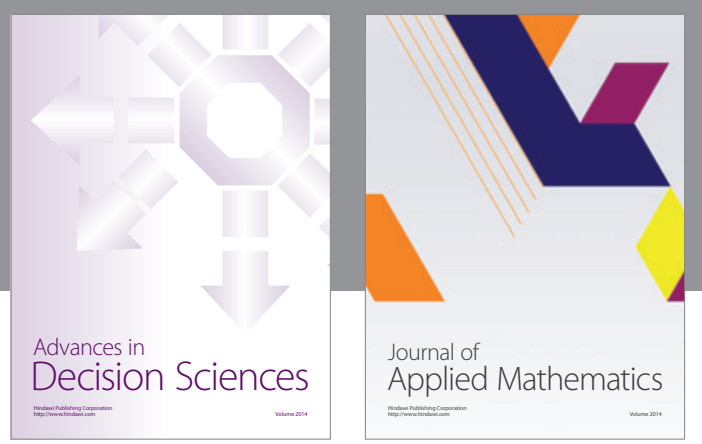

Algebra

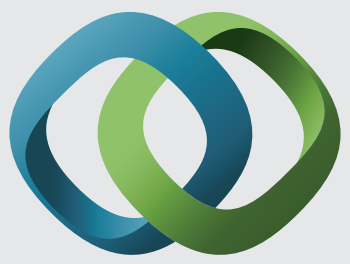

\section{Hindawi}

Submit your manuscripts at

http://www.hindawi.com
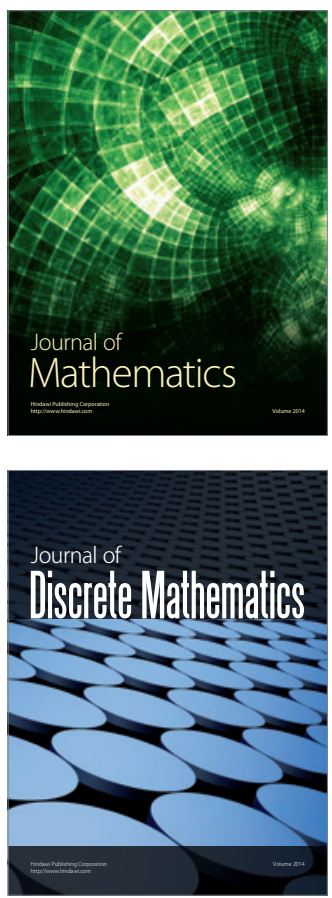

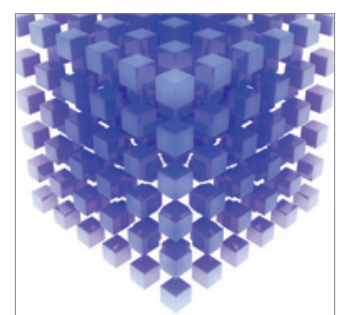

Mathematical Problems in Engineering
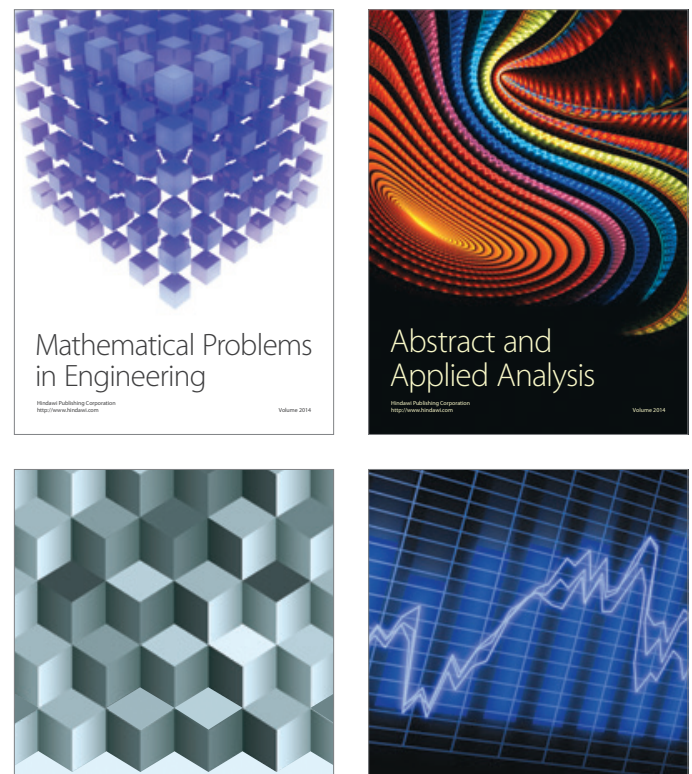

Journal of

Function Spaces

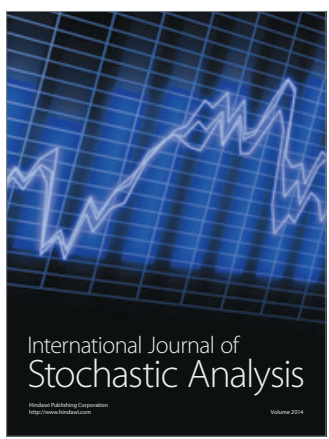

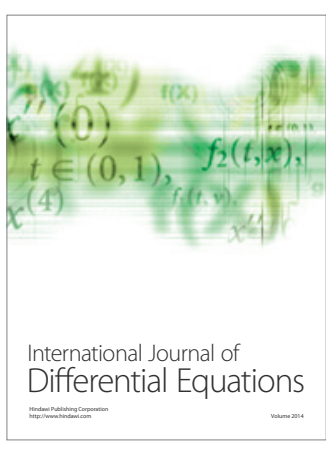
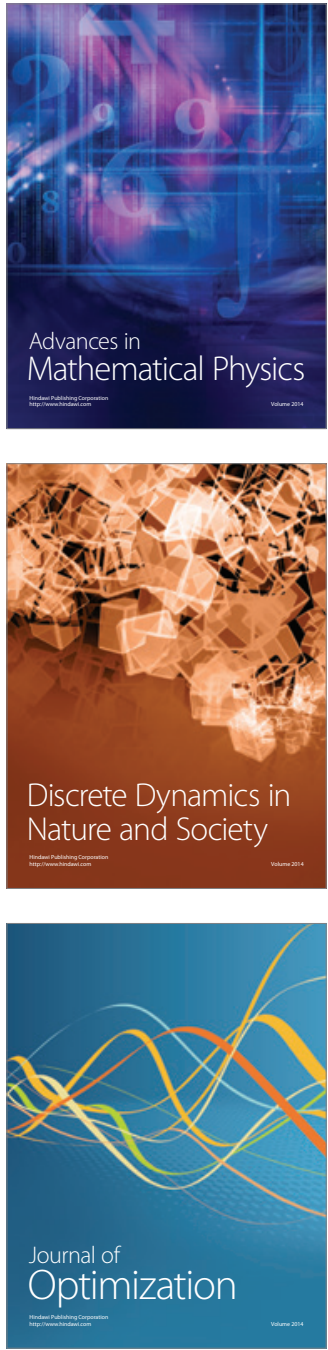